\title{
Proteomic analysis of insulin secretory granules in INS-1 cells by protein correlation profiling
}

\author{
Min $\mathrm{Li}^{1,2}$, Wen Du${ }^{1}$, Maoge Zhou ${ }^{1}$, Li Zheng ${ }^{1}$, Eli Song ${ }^{1 凶}$, Junjie Hou ${ }^{1 凶}$ \\ ${ }^{1}$ National Laboratory of Biomacromolecules, CAS Center for Excellence in Biomacromolecules, Institute of \\ Biophysics, Chinese Academy of Sciences, Beijing 100101, China \\ ${ }^{2}$ College of Life Sciences, University of Chinese Academy of Sciences, Beijing 100049, China
}

Received: 12 April 2018 / Accepted: 8 July 2018 / Published online: 29 August 2018

\section{Graphical abstract}

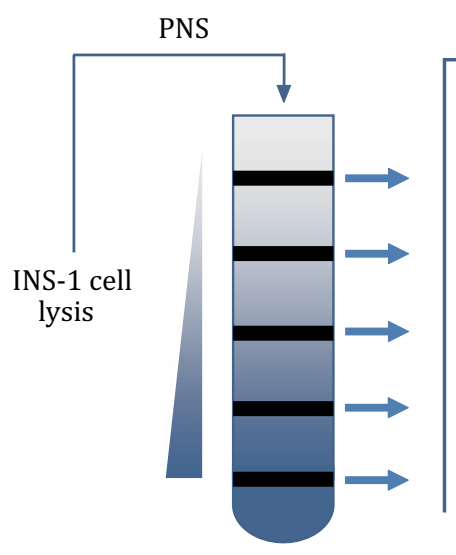

OptiPrep density
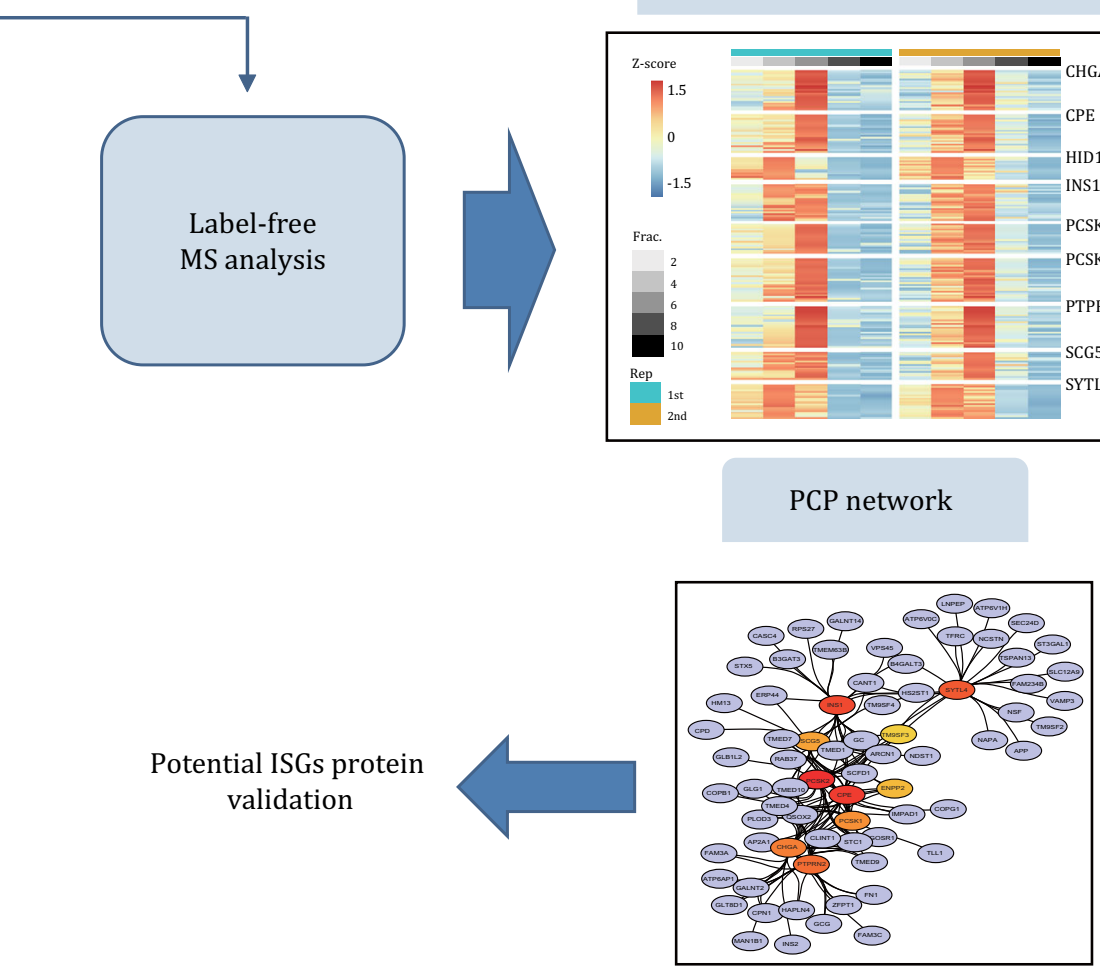

Electronic supplementary material The online version of this article (https://doi.org/10.1007/s41048-018-0061-3) contains supplementary material, which is available to authorized users.

$\triangle$ Correspondence: songali@ibp.ac.cn (E. Song), houjunjie@moon.ibp.ac.cn (J. Hou) 
Abstract Insulin secretory granules (ISGs), a group of distinguishing organelles in pancreatic $\beta$ cells, are responsible for the storage and secretion of insulin to maintain blood glucose homeostasis. The molecular mechanisms of ISG biogenesis, maturation, transportation, and exocytosis are still largely unknown because the proteins involved in these distinct steps have not been fully identified. Subcellular fractionation by density gradient centrifugation has been successfully employed to analyze the proteomes of numerous organelles. However, use of this method to elucidate the ISG proteome is limited by co-fractionated contaminants because ISGs are very dynamic and have abundant exchanges or contacts with other organelles, such as the Golgi apparatus, lysosomes, and endosomes. In this study, we developed a new strategy for identifying ISG proteins by protein correlation profiling (PCP)-based proteomics, which included ISG purification by OptiPrep density gradient centrifugation, label-free quantitative proteome, and identification of ISG proteins by correlating fractionation profiles between candidates and known ISG markers. Using this approach, we were able to identify 81 ISG proteins. Among them, TM9SF3, a nine-transmembrane protein, was considered a high confidence ISG candidate protein highlighted in the PCP network. Further biochemical and immunofluorescence assays indicated that TM9SF3 localized in ISGs, suggesting that it is a potential new ISG marker.

Keywords Insulin secretory granules (ISGs), Proteome, Label-free proteomics, Protein correlation profiling

\section{INTRODUCTION}

Insulin plays a crucial role in regulating energy balance and blood glucose homeostasis. Most insulin is synthesized, stored, and secreted by pancreatic $\beta$ cells (Straub et al. 2004). In $\beta$ cells, insulin and other secretory peptides are stored in a specialized organelle, namely, insulin secretory granules (ISGs), which serve as a storage pool. When the blood glucose level increases, ISGs fuse with the plasma membrane and release insulin. ISG biogenesis starts with budding at the transGolgi network (TGN), where proinsulin and other prohormones are sorted into immature insulin secretory granules (iISGs) (Arvan and Castle 1998; Arvan and Halban 2004). Then, iISGs undergo a precisely controlled maturation process to become mature ISGs (mISGs). The maturation process consists of several distinct steps, including homotypic fusion of iISGs, acidification of the granular lumen, proteolytic processing of prohormones into mature hormones, condensation of cargoes and removal of unwanted membrane and proteins. The resulting mISGs will be subsequently stored in a ready release pool or transported to the vicinity of the cell membrane, exhibiting specific responses to stimuli for regulated exocytosis (Du et al. 2016; Schvartz et al. 2012). However, due to lack of the sufficient understanding about the proteins involved in ISG-associated processes, including biogenesis, maturation, transportation, and exocytosis, the molecular mechanisms of these events are still largely unknown (Katsumata-Kato et al. 2015).

The map of organelle proteome is commonly achieved by subcellular fractionation methods, such as density gradient centrifugation and fluorescent-assisted organelle sorting (Lee et al. 2010). However, for ISGs, accurate proteome identification is a major challenge because ISGs are highly dynamic and have abundant exchanges or contacts with other organelles. Several studies have attempted to characterize the ISG proteome through proteomic analysis of ISG-enriched, biochemically isolated subcellular fractions. Brunner et al. (2007) identified 130 ISG proteins by using a two-step gradient purification of ISGs from INS-1E cells. In another study, Schvartz et al. (2012) used a three-step gradient purification procedure combined with stable isotope labeling with amino acids in cell culture (SILAC) to identify 140 proteins in mISGs. In addition, Hickey et al. (2009) employed density gradient centrifugation and fractionation and a further immuno-affinity approach, using antibodies specific to Vamp2 (vesicle-associated membrane protein 2), to enrich ISGs and finally identified 51 proteins in INS-1E cells. However, it was difficult to distinguish ISGs based on the size and the density of the granules by these analytical separation methods in previous studies (Tooze et al. 1991); thus, the interpretation of the proteomic data has been complicated by the presence of contaminant proteins from co-isolated organelles and/or membrane fragments, such as the Golgi apparatus, lysosomes and endosomes.

Protein correlation profiling (PCP)-based proteomics is an advantageous analytical method to identify multiprotein complexes or subcellular organelles, which are enriched by fractionation but not purified to homogeneity (Andersen et al. 2003; Kristensen et al. 2012). The basic principle of PCP analysis is to calculate the degree of similarity between the abundance profiles of 
candidate proteins with a "consensus profile", defined as the average of the profiles of known marker proteins. A candidate with a high degree of similarity tends to be a protein of interest with strong potential. Recently, PCP studies have been successfully applied to investigate some subcellular organelles (Andersen et al. 2002, 2005; Foster et al. 2006; Krahmer et al. 2013), such as nucleolar and lipid droplets, but this approach has not previously been applied to ISGs.

We previously established an efficient two-step subcellular fractionation method for the enrichment of insulin granules from INS-1 cells, which involved OptiPrep gradient purification followed by Percoll solution purification. Using this method, the iISGs and mISGs can be successfully distinguished and enriched (Chen et al. 2015). In this study, we first separated the post-nuclear supernatant into five subcellular fractions by OptiPrep gradient centrifugation, and each fraction was then separated by SDS-PAGE, followed by in-gel digestion and mass spectrometry (MS) analysis. Purification profiles from ISG marker proteins quantified by MS intensity were used to define the consensus curves. Euclidean distance (ED) between the consensus curves and PCPs of all identified proteins was calculated and sorted. Finally, we considered 81 proteins in the top 100 ranked list that were identified repeatedly in both biological experiments as the ISG proteins. By constructing a PCP network analysis, TM9SF3, a 9-transmembrane protein, was highlighted as a high confidence ISG candidate protein. Further biochemical and immunofluorescence assays demonstrated that TM9SF3 co-localized with insulin, suggesting that it has the potential to be a new ISG marker.

\section{EXPERIMENTAL}

\section{Materials and reagents}

OptiPrep $^{\mathrm{TM}}$ Density Gradient Medium, $\mathrm{MgSO}_{4}$, EDTA, HEPES, MES, and KOH were purchased from Sigma (St. Louis, MO, USA). EGTA was purchased from Amresco (USA). Sucrose was purchased from Beijing Biodee Biotechnology (Beijing, China).

The following antibodies were used for immunofluorescence and Western blot analysis: mouse monoclonal anti-proinsulin was purchased from HyTest (Turku, Finland). Guinea pig polyclonal antibody against insulin and rabbit monoclonal anti-TOMM20 antibody were purchased from Abcam (Cambridge, MA). Rabbit polyclonal anti-Vamp4, rabbit polyclonal anti-ATP6V1H, rabbit polyclonal anti-HID-1, rabbit polyclonal anti-CANX, rabbit polyclonal anti-CPE, rabbit polyclonal anti-SYTL4, and rabbit polyclonal anti-STX6 antibodies were purchased from Proteintech (Wuhan, China). Rabbit polyclonal antiTM9SF3 antibody was purchased from Invitrogen (Carlsbad, CA, USA). Rabbit polyclonal anti-TM9SF3 antibody was purchased from Sigma (St. Louis, MO, USA).

\section{Cell culture}

The INS-1 cell line was obtained from the Cell Resource Center, Peking Union Medical College (the headquarters of the National Infrastructure of Cell Line Resource, NSTI, Beijing, China) and was cultured in 1640 medium supplemented with $10 \%$ fetal bovine serum (Gibco 10270, South America), $1 \mathrm{mmol}$ penicillinstreptomycin, and $100 \mu \mathrm{mol}$ mercaptoethanol at $37{ }^{\circ} \mathrm{C}$ with $5 \% \mathrm{CO}_{2}$.

\section{Optiprep separation of post-nuclear supernatant (PNS) from INS-1 cells}

Separation of PNS was performed as described in a previous study (Chen et al. 2015) with minor modifications. In brief, INS-1 cells were grown on 15-cmdiameter dasher plate and washed twice with ice-cold PBS. Cells were harvested by trypsin and centrifuged at $1500 \mathrm{~g}$ for $10 \mathrm{~min}$ at $4{ }^{\circ} \mathrm{C}$. The cell pellet was suspension with $2 \mathrm{ml}$ homogenization buffer $(0.3 \mathrm{~mol} / \mathrm{L}$ sucrose, $1 \mathrm{mmol} / \mathrm{L}$ EDTA, $1 \mathrm{mmol} / \mathrm{L} \mathrm{MgSO}_{4}, 10 \mathrm{mmol} / \mathrm{L}$ MES-KOH, pH 6.5) containing 1:100 protease inhibitor (PI) by nitrogen cavitation for $20 \mathrm{~min}$ at $500 \mathrm{psi}$ on ice. The optimized centrifugal speed was from 1000 to $5000 \mathrm{~g}$. The cell lysis procedure not only removed unbroken cells and nuclear debris but also cell organelles and large complexes from the cell lysate by centrifuging at $5000 \mathrm{~g}$ for $15 \mathrm{~min}$. The cell supernatant was collected and loaded on top of a discontinuous Optiprep gradient composed of five layers. The OptiPrep density gradient included five concentrations from top to bottom $(8.8 \%, 13.2 \%, 17.6 \%, 23.4 \%, 30 \%)$, and the volume of each layer was $2 \mathrm{ml}$. Then, 30\% OptiPrep was diluted with Buffer B (2 mmol/L EGTA, $20 \mathrm{mmol} / \mathrm{L} \mathrm{MES-KOH,}$ $\mathrm{pH}$ 6.5), and the remaining solution was obtained by diluting 30\% OptiPrep with Buffer A containing 1:100 PI in a SW40 tube. The sample was centrifuged at $100,000 \mathrm{~g}$ for $135 \mathrm{~min}$, and the enrichment fraction was collected, which was diluted with Buffer A and centrifuged at $12,000 \mathrm{~g}$ for $15 \mathrm{~min}$. The pellet was collected and washed three times with Buffer A.

\section{In-gel protein digestion}

Subcellular fractions 2, 4, 6, 8, and 10 were separated on a 12\% SDS-PAGE gel and stained overnight with Coomassie G-250 (Invitrogen). Lanes of each fraction were 
manually cut into three gel bands. In-gel protein digestion was performed as described in the literature (Zhu et al. 2018). The tryptic peptides were dried using a SpeedVac and stored at $-20{ }^{\circ} \mathrm{C}$ for further analysis.

\section{MS analysis}

The nano Liquid chromatography (LC)-MS/MS experiments were performed on a $\mathrm{Q}$ Exactive mass spectrometer (Thermo Scientific) coupled to an Easy-nLC 1000 HPLC system (Thermo Scientific). The dried peptides were resuspended in $0.1 \%$ formic acid $/ 2 \%$ acetonitrile, and loaded onto a $100 \mu \mathrm{m}$ id $\times 2 \mathrm{~cm}$ fused silica trap column packed in-house with reversed phase silica (Reprosil-Pur C18 AQ $5 \mu \mathrm{m}$, Dr. Maisch GmbH) and then separated on an a $75 \mu \mathrm{m}$ id $\times 20 \mathrm{~cm} \mathrm{C18}$ column packed with reversed phase silica (Reprosil-Pur C18 AQ $3 \mu \mathrm{m}$, Dr. Maisch GmbH). The loaded peptides were eluted with a 78-min gradient. The solvent A consisted of $0.1 \% \mathrm{FA}$ in water solution and the solvent $\mathrm{B}$ consisted of $0.1 \% \mathrm{FA}$ in acetonitrile solution. The segmented gradient was $4 \%-12 \% \mathrm{~B}, 5 \mathrm{~min} ; 12 \%-22 \% \mathrm{~B}$, $50 \mathrm{~min} ; 22 \%-32 \%$ B, $12 \mathrm{~min} ; 32 \%-95 \%$ B, 1 min; 95\% $\mathrm{B}, 7 \mathrm{~min}$ at a flow rate of $280 \mathrm{nl} / \mathrm{min}$.

The mass spectrometer was operated in the datadependent acquisition mode, and full-scan MS data were acquired in the Orbitrap with a resolution of 70,000 $(\mathrm{m} / \mathrm{z} 200)$ across the mass range of $300-1600 \mathrm{~m} / \mathrm{z}$. The target value was $3.00 \times 10^{6}$ with a maximum injection time of $60 \mathrm{~ms}$. After the survey scans, the top 20 most intense precursor ions were selected for MS/MS fragmentation with isolation width of $2 \mathrm{~m} / z$ in the HCD collision cell with optimized normalized collision energy of $32 \%$. Subsequently, MS/MS spectra were acquired in the Orbitrap with a resolution of $17,500(\mathrm{~m} / \mathrm{z} 200)$ and a low mass cut-off setting of $100 \mathrm{~m} / \mathrm{z}$. The target value was set as $5.00 \times 10^{4}$ with a maximum injection time of $80 \mathrm{~ms}$. The dynamic exclusion time was $50 \mathrm{~s}$. For nanoelectrospray ion source setting, the spray voltage was $2.0 \mathrm{kV}$; no sheath gas flow; the heated capillary temperature was $320^{\circ} \mathrm{C}$.

The raw MS data were processed with Maxquant (v1.5.0.30). The UniProt Rattus norvegicus proteome supplemented with all the frequently observed contaminants in MS served as the database. To search precursor and fragment ions, an initial maximal mass deviation of 10 and $20 \mathrm{ppm}$, respectively, was required. Tryptic full enzyme specificity with no proline restriction and only peptides with a minimum length of seven amino acids were selected. A maximum of two missed cleavages were allowed. Carbamidomethylation (Cys) was set as the fixed modification. Oxidation (Met) and $\mathrm{N}$-acetylation were considered variable modifications.
For identification of protein and peptide, we required a maximum FDR of $1 \%$.

\section{Western blot}

The pellets of enriched granules were prepared in RIPA buffer $(50 \mathrm{mmol} / \mathrm{L}$ Tris pH 7.4, $150 \mathrm{mmol} / \mathrm{L} \mathrm{NaCl}, 0.1 \%$ SDS, $0.5 \%$ sodium deoxycholate, $1 \%$ Triton X-100, protease cocktail, $1 \mathrm{mmol} / \mathrm{L}$ PMSF, $10 \mathrm{mmol} / \mathrm{L}$ sodium azide, $10 \mathrm{mmol} / \mathrm{L}$ sodium ascorbate, and $5 \mathrm{mmol} / \mathrm{L}$ Trolox) for $20 \mathrm{~min}$ on the ice. After centrifugation to remove insoluble materials, the protein concentration was determined with the bicinchoninic acid assay (Thermo Scientific, 23227). The sample was diluted with $5 \times$ SDS buffer for $10 \mathrm{~min}$ at $95{ }^{\circ} \mathrm{C}$, separated by SDSPAGE and transferred to a nitrocellulose membrane at $400 \mathrm{~mA}$ for $60 \mathrm{~min}$ in a $10 \%$ methanol transfer buffer. The membrane was first blocked for $1 \mathrm{~h}$ with $5 \%$ non-fat dry milk in TBST and then incubated with the primary antibody overnight at $4{ }^{\circ} \mathrm{C}$. The membrane was washed six times for 5 min each time in TBST, then incubated with the second IgG antibodies conjugated with horseradish peroxidase (HRP) for $1 \mathrm{~h}$ at the room temperature, and washed six times for 5 min each time in TBST. Western blotting signals were detected and analyzed using HRP and ChemiScope Touch 6000 (Clinx, Shanghai, China).

\section{Immunofluorescence}

INS-1 cells grown in a glass bottom dish were washed in PBS, then fixed in $4 \%$ paraformaldehyde (PFA) for 20 min and permeabilized with $0.2 \%$ saponin and $5 \%$ albumin bovine in PBS for $1 \mathrm{~h}$ at room temperature. Cells were washed two times for 5 min each time and incubated with the primary antibody overnight at $4{ }^{\circ} \mathrm{C}$. After washing six times for 5 min each time, the cells were labeled with secondary antibodies for $1 \mathrm{~h}$ (insulin was labeled by TRITC-conjugated goat anti-guinea pig, while TM9SF3 was labeled by Alexa Fluor 488-conjugated goat anti-rabbit). Then, the cells were washed six times for 5 min each time and mounted with DAPI Fluoromount-G. All confocal microscope images were generated using an Olympus FV1200 Laser Scanning Confocal Microscope (Olympus, Tokyo, Japan) with a $100 \times(\mathrm{NA}=1.40)$ oil objective.

\section{RESULTS AND DISCUSSION}

We reported a new strategy for efficient identification of the ISG proteome in INS-1 cells. As shown in Fig. 1A, this strategy includes three steps: (1) enrichment of the ISG fraction by OptiPrep density gradient centrifugation; 


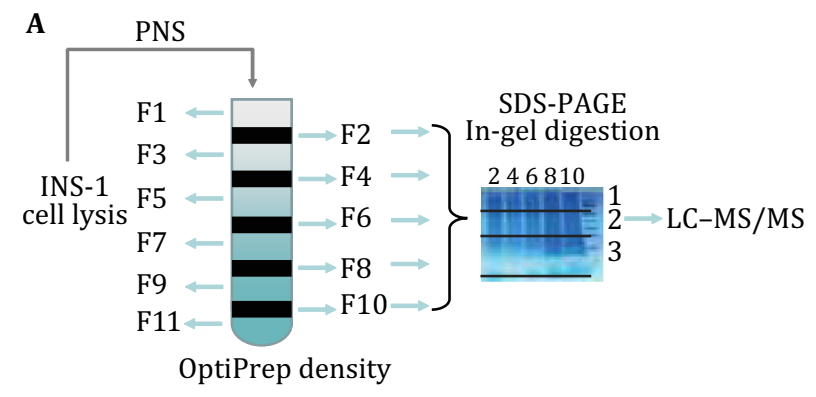

B

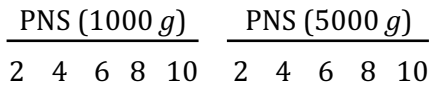

TOMM20 - - $-10=-$

C

OptiPrep fraction

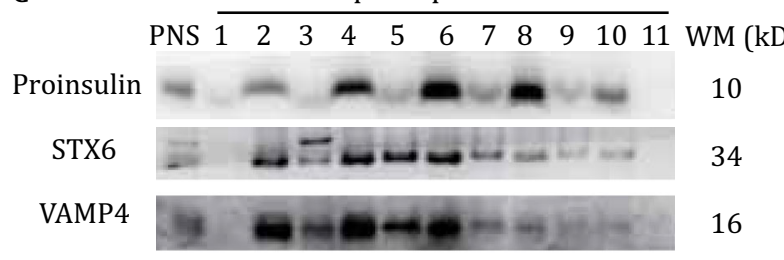

Fig. 1 A Post-nuclear supernatant separation with OptiPrep density gradient centrifugation, followed by SDS-PAGE, in-gel protein digestion, and peptide analysis by LC-MS/MS. B Western blot analysis of TOMM20 in OptiPrep fractions of PNS collected with centrifugation speeds of 1000 and $5000 \mathrm{~g}$. C Western blot analysis of proinsulin, STX6, and VAMP4 in each OptiPrep fraction. All antibodies were diluted 1:1000

(2) protein separation by SDS-PAGE followed by in-gel protein digestion and LC-MS/MS analysis; and (3) PCP analysis.

For the enrichment of the ISG fraction, INS-1 cells were lysed and centrifuged at $1000 \mathrm{~g}$. The pellets were resuspended and further centrifuged at $5000 \mathrm{~g}$ to remove most of the mitochondrial fractions or other heavy subcellular organelles. The resulting PNS fraction contained less mitochondria than that prepared by low centrifugation speed, as shown by detection of the mitochondrial marker protein TOMM20 (Fig. 1B). Next, a discontinuous OptiPrep density gradient that had five concentrations $(8.8 \%, 13.2 \%, 17.6 \%, 23.4 \%$, and $30 \%)$ (Chen et al. 2015) was prepared for the separation of PNS. All resulting twelve fractions and raw PNS were assessed by Western blotting experiments to measure the efficiency of OptiPrep separation. As shown in Fig. 1C, proinsulin was mainly detected in fractions 4,6 , and 8. Syntaxin 6 (STX6), which mediates iISG homotypic fusion (Wendler et al. 2001), was abundant in fractions 4 and 6 . Vesicle-associated membrane protein 4 (VAMP4), which was implicated in TGN vesicle trafficking (Steegmaier et al. 1999), was mainly enriched in fractions 2, 4, and 6 and rarely detected in fractions 8 and 10 . These results indicated that the ISG proteins were enriched in fractions 4,6 , and 8 , and iISG proteins were mainly in fractions 4 and 6 .

To identify membrane proteins more efficiently, the proteins in each OptiPrep fraction were identified by the use of in-gel digestion-based proteomics workflow (Choksawangkarn et al. 2012). All the MS raw data were analyzed by Maxquant software (Cox and Mann 2008). With a false discovery rate of less than $1 \%$ at both the peptide and protein levels, 2070 proteins were repeatedly identified by both biological experiments. MS intensity of proteins/protein groups reported by Maxquant was used as the relative quantitative abundance of proteins. To assess the reproducibility of the proteomics experiments, we performed Pearson correlation analysis based on the results of hierarchical clustering of proteins quantified in each OptiPrep fraction. The results showed that proteins in same fraction from two independent experiments were clustered together (Fig. 2A), indicating that our quantitative proteome was

A

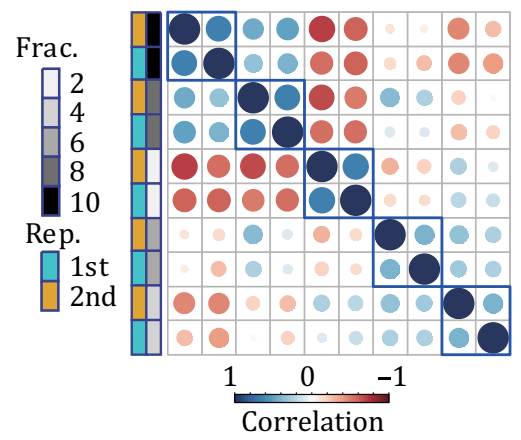

B

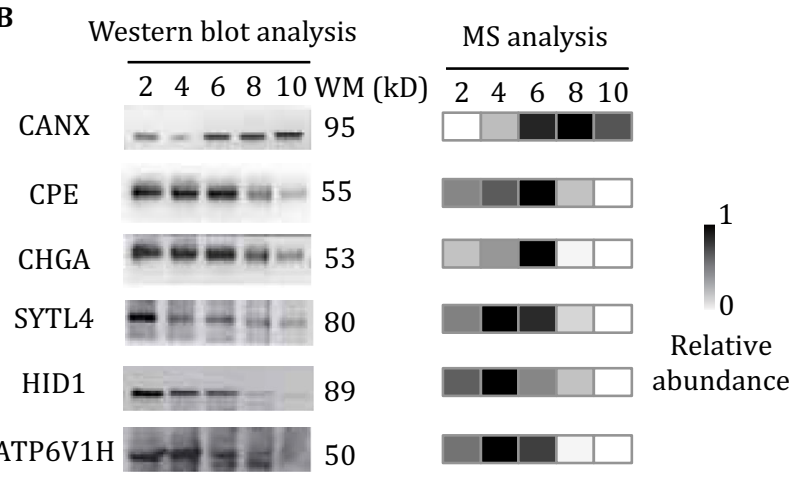

Fig. 2 A Pearson correlation matrix based on the results of hierarchical clustering of proteins quantified in each OptiPrep fraction by two independent experiments, and blue rectangles indicate the reproducibility of biological replicates. B Six proteins (CANX, CPE, CHGA, SYTL4, HID-1, and ATP6V1H) were randomly selected for the comparison of their relative abundances detected by Western blot and MS in OptiPrep fractions 2, 4, 6, 8, and 10. All antibodies were diluted 1:1000 


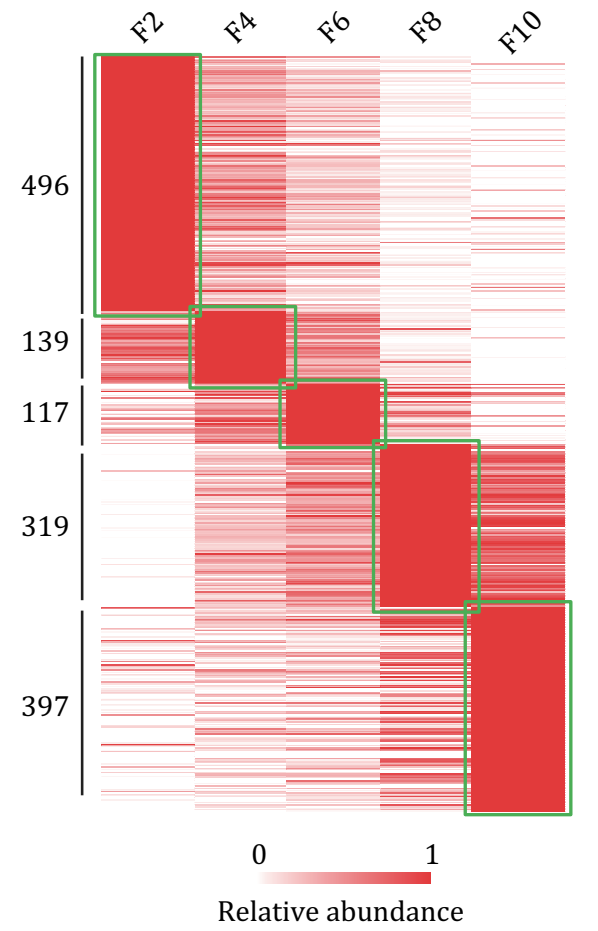

Enriched GO_CC_DIRECT terms (DAVID) $p$-value

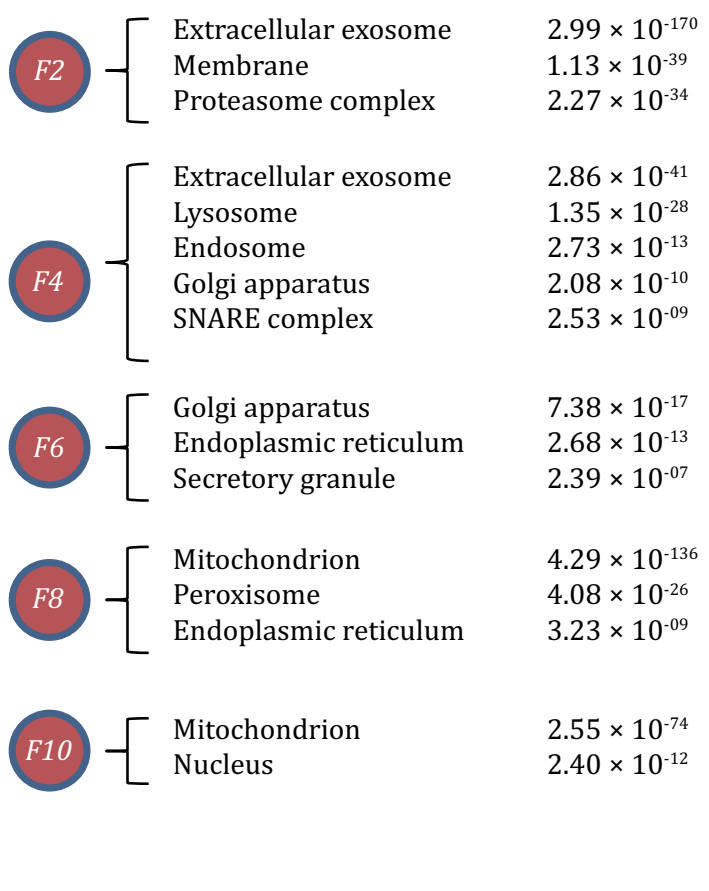

Fig. 3 The heatmap of normalized PCP indicating the enrichment of proteins in each OptiPrep fraction. GO cellular component annotation analysis was performed with the DAVID tool. Representative enriched GO terms and their $p$ value are listed

highly reproducible. We next performed Western blots to analyze several randomly selected proteins, including CANX, CPE, CHGA, SYTL4, HID-1, and ATP6V1H (Fig. 2B). The results showed that the relative abundance of proteins measured by MS and Western blot was quite comparable, demonstrating the high accuracy of our quantitative proteomic data.

Next, we refined the proteome data by excluding the proteins in which the relative abundance correlation between two replicates was less than 0.5. We identified 1468 proteins as the high confidence proteome data for the next PCP analysis. The MS intensities of proteins detected by two replicates were averaged and normalized using the max-min normalization method as quantitative PCP dataset. To analyze the general feature of the proteome in each subcellular fraction, we categorized the proteins based on their PCP distributions into five groups; 496, 139, 117, 319, and 397 proteins with clearly similar abundance patterns were highly enriched in OptiPrep fractions 2, 4, 6, 8, and 10, respectively (Fig. 3). The GO cellular component annotation analysis by DAVID (da Huang et al. 2009) revealed that fraction 2 mainly comprised proteins from the extracellular exosome, membrane, and proteasome; fraction 4 mainly comprised proteins from the lysosome, endosome, Golgi apparatus, and SNARE complex; fraction 6 was enriched in proteins from organelles such as the Golgi apparatus, endoplasmic reticulum (ER), and secretory granule; fraction 8 was highly enriched in proteins from the mitochondria, peroxisome and ER, and fraction 10 mainly comprised proteins from the mitochondria and nucleus. Interestingly, we found that ISG-related proteins were highly enriched in fraction 6, including CHGA, PTPRN2, INS1, INS2, PAM, PCSK2, and RAB37, indicating our subcellular fractionation method successfully enriched ISGs in one fraction.

For PCP analysis of ISG, the ideal marker proteins of ISGs are proinsulin and insulin, representing iISGs and mISGs, respectively. However, bottom-up-based proteomics techniques cannot distinguish proinsulin, insulin, and C-peptide after tryptic protein digestion because of the common tryptic peptides (Cheng et al. 2015). So our MS-measured insulin represented the total amount of protein products encoded by Ins1 and cannot be used as a reference to dissect the iISGs and mISGs. To address such limitation, we selected another eight known ISGrelated proteins, including CHGA (Bandyopadhyay and Mahata 2017), CPE (Cool and Loh 1998; Rindler 1998), HID1 (Du et al. 2016), PCSK1 (Furukawa et al. 1999), 
$\mathbf{A}$

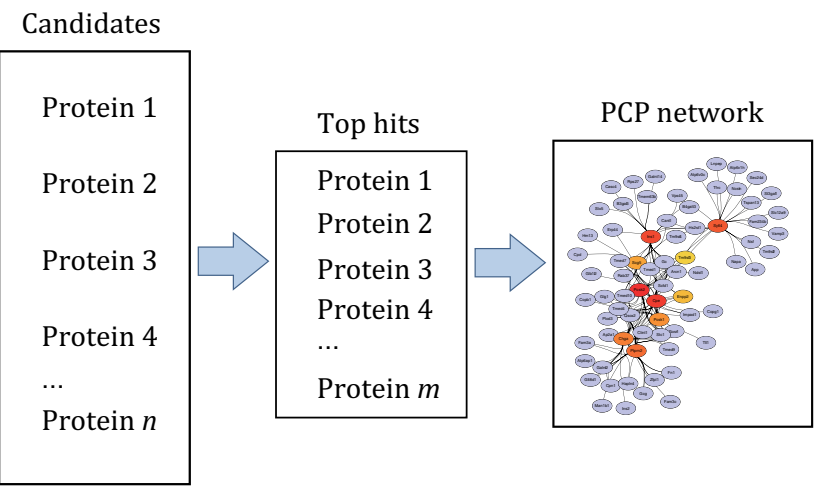

B

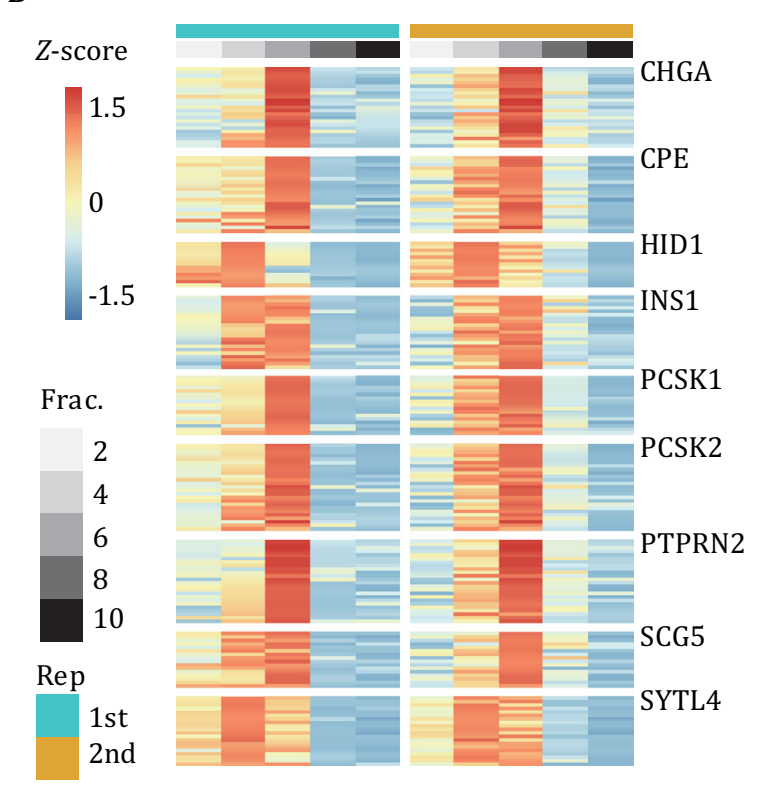

C

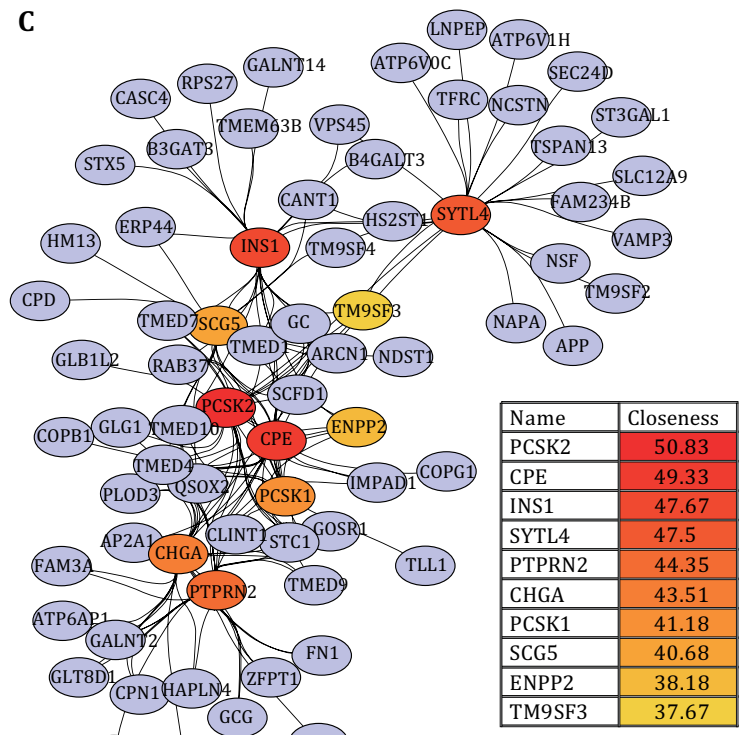

D

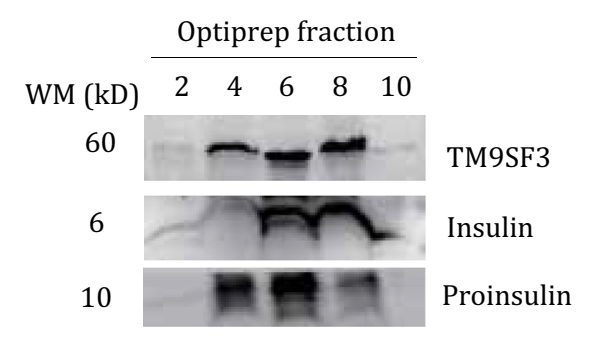

$\mathbf{E}$

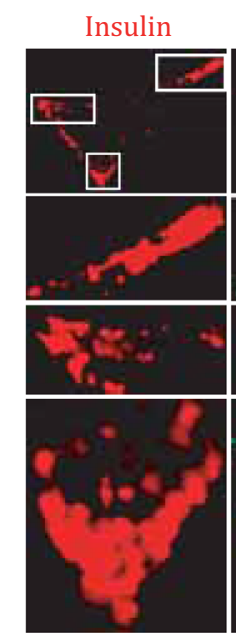

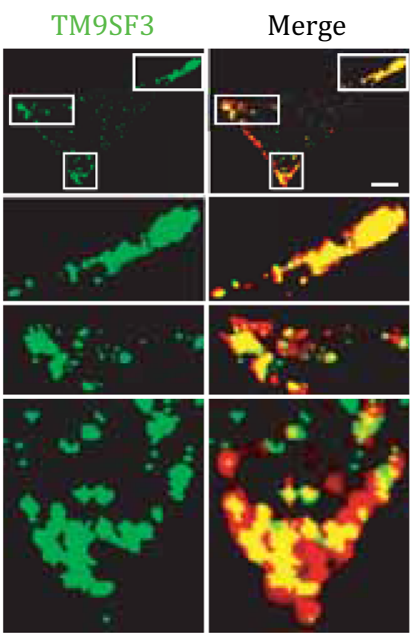

Fig. 4 A Schematic to illustrate the PCP data analysis. B Heatmap of relative abundance of potential ISG proteins. Data were scaled by Z-transformation. C PCP network of potential ISG proteins. D Western blot analysis of TM9SF3, insulin and proinsulin in OptiPrep fractions. E Immunofluorescence micrographs showed the co-localization of TM9SF3 (green) and insulin (red) in INS-1 cells. Anti-insulin was diluted 1:400, and anti-TM9SF3 was diluted 1:100. Scale bar: $5 \mu \mathrm{m}$ 
PCSK2 (Guest 2017), PTPRN2 (Caromile et al. 2010; Suckale and Solimena 2010), SCG5 (Osamura et al. 1988; Tatsumi et al. 2003) and SYTL4 (Torii et al. 2002; Yi et al. 2002). These proteins participated in the distinct steps of ISG maturation process representing the dynamic composition of ISG, so we treated them as a group-marker of ISGs to perform PCP analysis as illustrated in Fig. 4A. ED of purification profiles between the group-marker and each quantified proteins was calculated iteratively. The top 100 lists ranked by ED were recognized as the significant candidates (Supplemental Tables S1, S2). Finally, 81 ISG candidate proteins were repeatedly identified in two biological replicates (Fig. 4B). The PCP network was further built to visualize the relationship between the group-marker and ISG candidates (Fig. 4C). The top ten nodes ranked by the closeness score of the PCP network were highlighted to demonstrate the central nodes. Except for HID-1, all other proteins and the group-marker were top-ranked, indicating the rationality of selecting them as ISG groupmarkers.

Among 81 ISG proteins identified in our study, several proteins are known ISG-related proteins, including Insulin1/2, CPE, PCSK1, PCSK2, CHGA, ATP6AP1, (Supplemental Tables S1, S2), suggesting the efficiency of our strategy and the reliability of our dataset. During ISG biogenesis, maturation, transportation, and exocytosis, membrane proteins are considered to play essential roles. In our data, more than $60 \%$ identified proteins are membrane proteins or membrane-associated proteins. Some of them are potential to function in ISG-related processes, such as ARFRP1, SCFD1, STX12, STX5, VPS45, and ATP6V0C. For example, ATP6V0C is an essential subunit of vacuolar ATPase, a critical enzyme complex that regulates the acidification of the granular lumen which is one of the indispensable steps during ISG maturation (Mangieri et al. 2014). SNARE- and SNAREassociated proteins are critical factors for mediating membrane fusion (Advani et al. 1998; Laufman et al. 2009; Zouhar et al. 2009). During ISG maturation, immature ISGs need undergo a homotypic fusion process, in which several SNARE proteins, such as STX12, VTI1A, STX6, and VAMP4, were reported to be involved (Brandhorst et al. 2006; Zwilling et al. 2007). We speculated STX12 may also play a role in regulating ISG maturation. Taken together, our results suggested that, compared with conventional subcellular fraction method, the PCP-based strategy is more effective for the identification of ISG proteins.

Intriguingly, we noticed that a membrane protein, TM9SF3, was included in the top 10 lists of central nodes (Fig. 4C). For verification, we first determined the protein abundance of TM9SF3 in each OptiPrep fraction. Compared with the distribution of proinsulin and insulin, TM9SF3 distribution was well correlated with insulin content (Fig. 4D). Next, we investigated the cellular distribution of TM9SF3 in INS-1 cells (Fig. 4E). Immunofluorescence results showed that TM9SF3 had good co-localization with insulin, especially in the cell periphery. These results indicated that TM9SF3 may be a new ISG maker. More extensive studies are needed in the future to examine whether TM9SF3 has functions in ISG maturation.

The PCP-based strategy used in the present work can be applicable for ISG functional research by defining the dynamic ISG proteome under different physiological conditions. Furthermore, it can be easily adapted for the proteomic analysis of other highly dynamic organelles, such as endosomes. Our study demonstrated that labelfree proteomics was also compatible with PCP analysis, just as other labeling-based methods, such as isobaric labeling and SILAC. Therefore, one can adopt the same strategy to investigate the dynamic proteomes in organelles under multiple stimuli. Since there is no need for labeling protein/peptide, our PCP strategy can be used to identify the organelle proteome from a wide range of sources, including cells and tissues.

\section{CONCLUSION}

In this study, combined with subcellular fractionation by OptiPrep density gradient centrifugation, we employed a quantitative proteomics approach based on PCP analysis to efficiently measure the ISG proteome. This combination of optimized fractionation and PCP methodology enabled us to extract 81 potential ISG proteins from thousands of co-isolated proteins with high confidence. Several previously known ISG proteins were included in our lists, validating our approach. Further data mining by PCP network analysis enabled us to find and validate a new potential ISG protein, TM9SF3. This strategy can also be easily extended to identify the proteomes of other highly dynamic organelles, such as endosomes, and define the dynamic organelle proteome under different physiological conditions.

Acknowledgements This work was financially supported by Grants from Ministry of Science and Technology of the People's Republic of China (2016YFA0500203), the National Natural Science Foundation of China (31770900, 31730054).

\section{Compliance with Ethical Standards}

Conflict of interest Min Li, Wen Du, Maoge Zhou, Li Zheng, Eli Song, Junjie Hou declare that they have no conflict of interest. 
Human and animal rights and informed consent This article does not contain any studies with human or animal subjects performed by any of the authors.

Open Access This article is distributed under the terms of the Creative Commons Attribution 4.0 International License (http:// creativecommons.org/licenses/by/4.0/), which permits unrestricted use, distribution, and reproduction in any medium, provided you give appropriate credit to the original author(s) and the source, provide a link to the Creative Commons license, and indicate if changes were made.

\section{References}

Advani RJ, Bae HR, Bock JB, Chao DS, Doung YC, Prekeris R, Yoo JS, Scheller RH (1998) Seven novel mammalian SNARE proteins localize to distinct membrane compartments. J Biol Chem 273:10317-10324

Andersen JS, Lyon CE, Fox AH, Leung AK, Lam YW, Steen H, Mann M, Lamond AI (2002) Directed proteomic analysis of the human nucleolus. Curr Biol 12:1-11

Andersen JS, Wilkinson CJ, Mayor T, Mortensen P, Nigg EA, Mann M (2003) Proteomic characterization of the human centrosome by protein correlation profiling. Nature 426:570-574

Andersen JS, Lam YW, Leung AK, Ong SE, Lyon CE, Lamond AI, Mann M (2005) Nucleolar proteome dynamics. Nature 433:77-83

Arvan P, Castle D (1998) Sorting and storage during secretory granule biogenesis: looking backward and looking forward. Biochem J 332(Pt 3):593-610

Arvan P, Halban PA (2004) Sorting ourselves out: seeking consensus on trafficking in the beta-cell. Traffic 5:53-61

Bandyopadhyay GK, Mahata SK (2017) Chromogranin a regulation of obesity and peripheral insulin sensitivity. Front Endocrinol $8: 20$

Brandhorst D, Zwilling D, Rizzoli SO, Lippert U, Lang T, Jahn R (2006) Homotypic fusion of early endosomes: SNAREs do not determine fusion specificity. Proc Natl Acad Sci USA 103:2701-2706

Brunner Y, Coute Y, Iezzi M, Foti M, Fukuda M, Hochstrasser DF, Wollheim CB, Sanchez JC (2007) Proteomics analysis of insulin secretory granules. Mol Cell Proteomics 6:1007-1017

Caromile LA, Oganesian A, Coats SA, Seifert RA, Bowen-Pope DF (2010) The neurosecretory vesicle protein phogrin functions as a phosphatidylinositol phosphatase to regulate insulin secretion. J Biol Chem 285:10487-10496

Chen Y, Xia Z, Wang L, Yu Y, Liu P, Song E, Xu T (2015) An efficient two-step subcellular fractionation method for the enrichment of insulin granules from INS-1 cells. Biophys Rep 1:34-40

Cheng D, Zheng L, Hou J, Wang J, Xue P, Yang F, Xu T (2015) A new dimethyl labeling-based SID-MRM-MS method and its application to three proteases involved in insulin maturation. Biophys Rep 1:71-80

Choksawangkarn W, Edwards N, Wang Y, Gutierrez P, Fenselau C (2012) Comparative study of workflows optimized for in-gel, in-solution, and on-filter proteolysis in the analysis of plasma membrane proteins. J Proteome Res 11:3030-3034

Cool DR, Loh YP (1998) Carboxypeptidase E is a sorting receptor for prohormones: binding and kinetic studies. Mol Cell Endocrinol 139:7-13

Cox J, Mann M (2008) MaxQuant enables high peptide identification rates, individualized p.p.b.-range mass accuracies and proteome-wide protein quantification. Nat Biotechnol 26:1367-1372

da Huang W, Sherman BT, Lempicki RA (2009) Systematic and integrative analysis of large gene lists using DAVID bioinformatics resources. Nat Protoc 4:44-57

Du W, Zhou M, Zhao W, Cheng D, Wang L, Lu J, Song E, Feng W, Xue Y, Xu P, Xu T (2016) HID-1 is required for homotypic fusion of immature secretory granules during maturation. eLife 5:e18134

Foster LJ, de Hoog CL, Zhang Y, Zhang Y, Xie X, Mootha VK, Mann M (2006) A mammalian organelle map by protein correlation profiling. Cell 125:187-199

Furukawa H, Carroll RJ, Swift HH, Steiner DF (1999) Long-term elevation of free fatty acids leads to delayed processing of proinsulin and prohormone convertases 2 and 3 in the pancreatic beta-cell line MIN6. Diabetes 48:1395-1401

Guest PC (2017) Sequential immunoprecipitation of secretory vesicle proteins from biosynthetically labelled cells. Adv Exp Med Biol 974:157-165

Hickey AJ, Bradley JW, Skea GL, Middleditch MJ, Buchanan CM, Phillips AR, Cooper GJ (2009) Proteins associated with immunopurified granules from a model pancreatic islet beta-cell system: proteomic snapshot of an endocrine secretory granule. J Proteome Res 8:178-186

Katsumata-Kato O, Yokoyama M, Matsuki-Fukushima M, Narita T, Sugiya H, Fujita-Yoshigaki J (2015) Secretory proteins without a transport signal are retained in secretory granules during maturation in rat parotid acinar cells. Arch Oral Biol 60:642-649

Krahmer N, Hilger M, Kory N, Wilfling F, Stoehr G, Mann M, Farese RV Jr, Walther TC (2013) Protein correlation profiles identify lipid droplet proteins with high confidence. Mol Cell Proteomics 12:1115-1126

Kristensen AR, Gsponer J, Foster LJ (2012) A high-throughput approach for measuring temporal changes in the interactome. Nat Methods 9:907-909

Laufman 0, Kedan A, Hong W, Lev S (2009) Direct interaction between the COG complex and the SM protein, Sly1, is required for Golgi SNARE pairing. EMBO J 28:2006-2017

Lee YH, Tan HT, Chung MC (2010) Subcellular fractionation methods and strategies for proteomics. Proteomics 10:3935-3956

Mangieri LR, Mader BJ, Thomas CE, Taylor CA, Luker AM, Tse TE, Huisingh C, Shacka JJ (2014) ATP6V0C knockdown in neuroblastoma cells alters autophagy-lysosome pathway function and metabolism of proteins that accumulate in neurodegenerative disease. PLoS ONE 9:e93257

Osamura RY, Chretien M, Marcinkiewicz M (1988) Ultrastructural localization of secretory granule constituent chromogranin and 7 B 2. Pathol Res Pract 183:617-619

Rindler MJ (1998) Carboxypeptidase E, a peripheral membrane protein implicated in the targeting of hormones to secretory granules, co-aggregates with granule content proteins at acidic pH. J Biol Chem 273:31180-31185

Schvartz D, Brunner Y, Coute Y, Foti M, Wollheim CB, Sanchez JC (2012) Improved characterization of the insulin secretory granule proteomes. J Proteomics 75:4620-4631

Steegmaier M, Klumperman J, Foletti DL, Yoo JS, Scheller RH (1999) Vesicle-associated membrane protein 4 is implicated in trans-Golgi network vesicle trafficking. Mol Biol Cell 10:1957-1972

Straub SG, Shanmugam G, Sharp GW (2004) Stimulation of insulin release by glucose is associated with an increase in the number of docked granules in the beta-cells of rat pancreatic islets. Diabetes 53:3179-3183 
Suckale J, Solimena M (2010) The insulin secretory granule as a signaling hub. Trends Endocrinol Metab 21:599-609

Tatsumi KI, Tanaka S, Takano T, Tahara S, Murakami Y, Takao T, Hashimoto K, Kato Y, Teramoto A, Amino N (2003) Frequent appearance of autoantibodies against prohormone convertase $1 / 3$ and neuroendocrine protein $7 \mathrm{~B} 2$ in patients with nonfunctioning pituitary macroadenoma. Endocrine 22:335-340

Tooze SA, Flatmark T, Tooze J, Huttner WB (1991) Characterization of the immature secretory granule, an intermediate in granule biogenesis. J Cell Biol 115:1491-1503

Torii S, Zhao S, Yi Z, Takeuchi T, Izumi T (2002) Granuphilin modulates the exocytosis of secretory granules through interaction with syntaxin 1a. Mol Cell Biol 22:5518-5526

Wendler F, Page L, Urbe S, Tooze SA (2001) Homotypic fusion of immature secretory granules during maturation requires syntaxin 6. Mol Biol Cell 12:1699-1709
Yi Z, Yokota H, Torii S, Aoki T, Hosaka M, Zhao S, Takata K, Takeuchi $\mathrm{T}$, Izumi $\mathrm{T}$ (2002) The Rab27a/granuphilin complex regulates the exocytosis of insulin-containing dense-core granules. Mol Cell Biol 22:1858-1867

Zhu X, Li M, Xu X, Zhang R, Zhang X, Ma Z, Lu J, Xu T, Hou J, Song E (2018) hTAC internalizes via both clathrin-dependent and clathrin-independent endocytosis in mammalian cells. Protein Cell. https://doi.org/10.1007/s13238-018-0508-9

Zouhar J, Rojo E, Bassham DC (2009) AtVPS45 is a positive regulator of the SYP41/SYP61/VTI12 SNARE complex involved in trafficking of vacuolar cargo. Plant Physiol 149:1668-1678

Zwilling D, Cypionka A, Pohl WH, Fasshauer D, Walla PJ, Wahl MC, Jahn R (2007) Early endosomal SNAREs form a structurally conserved SNARE complex and fuse liposomes with multiple topologies. EMBO J 26:9-18 